\title{
The multi-time correlation functions, free white noise, and the generalized Poisson statistics in the low density limit
}

\author{
A. N. Pechen \\ Princeton University, Department of Chemistry, Frick Laboratory, Princeton, NJ \\ 08544-1009 \\ E-mail: apechen@princeton.edu
}

\begin{abstract}
In the present paper the low density limit of the non-chronological multitime correlation functions of boson number type operators is investigated. We prove that the limiting truncated non-chronological correlation can be computed using only a sub-class of diagrams associated to non-crossing pair partitions and thus coincide with the non-truncated correlation functions of suitable free number operators. The independent in the limit subalgebras are found and the limiting statistics is investigated. In particular, it is found that the cumulants of certain elements coincide in the limit with the cumulants of the Poisson distribution. An explicit representation of the limiting correlation functions and thus of the limiting algebra is constructed in a special case through suitably defined quantum white noise operators.
\end{abstract}




\section{INTRODUCTION}

The reduced dynamics of a quantum open system interacting with a reservoir in certain physical regimes is approximated by Markovian master equations. These regimes include the weak system-reservoir interactions and dilute reservoirs and in the theoretical framework they are described by certain limits. For a weakly interacting system one considers the limit as the coupling constant goes to zero (Weak Coupling Limit, WCL) whereas for a dilute reservoir one considers the limit as the density of the reservoir goes to zero (Low Density Limit, LDL) and an appropriate time rescaling should be performed in order to get a non-trivial limit. The Markovian reduced dynamics in these limits is considered in the review papers by Spohn and Lebowitz ${ }^{1,2}$. The reduced dynamics in the LDL was considered in details later by Dümcke ${ }^{3}$ using the method based on the quantum Bogoliubov-Born-Green-Kirkwood-Yvon hierarchy.

The total dynamics in these limits is governed by various quantum stochastic equations. There is a unique up to now approach, called the stochastic limit method, which allows an efficient derivation of the stochastic equations in the WCL. This approach is based on the quantum white noise technique and was developed by Accardi, $\mathrm{Lu}$, and Volovich ${ }^{4}$.

The convergence of the evolution operator of the total system in the LDL to a solution of a quantum stochastic equation was proved by Accardi and $\mathrm{Lu}^{5}$ and by Rudnicki, Alicki, and Sadowski ${ }^{6}$. Recently the low density limit was investigated with the quantum white noise technique ${ }^{7,8}$. This technique, well developed for the WCL, was non-trivially modified to include the LDL and for this case was called the stochastic golden rule for the low density limit. This technique was applied to the derivation of the quantum stochastic equations in the LDL. An advantage of the obtained equations is that they, in contrast with the exact Schrödinger equation, are explicitly solvable. At the same time they provide a good approximation of the exact dynamics.

The approach of ${ }^{7 / 8}$ uses the so called Fock-antiFock representation for the canonical commutation relations (CCR) algebra (this representation is unitary equivalent to the Gel'fand-Naimark-Segal representation). The difficulty with this approach is that the creation and annihilation operators in the Fock-antiFock Hilbert space do not describe creation and annihilation of physical particles and thus do not have direct physical meaning. To avoid this difficulty the investigation of the LDL directly in terms of the physical fields was performed ${ }^{9}$. Using this approach the chronological correlation functions in the LDL were found and the corresponding stochastic equations derived.

In the present paper we investigate the low density limit of the non-chronologically ordered correlation functions of boson number type operators. The investigation is related with $a b$ initio derivations of quantum stochastic equations describing quantum dynamics of a test particle interacting with a dilute gas. We find the limiting truncated correlation functions of the number type operators and show that they can be computed by representing the number operators through creation and annihilation operators and then considering only a sub-class of diagrams associated to non-crossing pair partitions. 
This fact allows to represent the limiting truncated correlation functions as the nontruncated correlation functions of number operators of a free quantum white noise thus making a connection with the Voiculescu free probability theory. We find the limiting statistics and show that the cumulants of certain elements coincide in the limit with the cumulants of the Poisson distribution.

The free probability theory was developed by Voiculescu around 1985 as a way to deal with von Neumann algebras of free groups. Then the theory was separated from this special context and began to develop as an independent field. In particular, applications of the free independence theory to random matrices were found. The details of free probability theory and its applications to random matrices could be found, for example, in references ${ }^{10 / 11}$.

Expectations of free random variables are characterized by diagrams associated to non-crossing pair partitions. The vanishing of crossing diagrams in the stochastic weak coupling limit for nonrelativistic QED and for the Anderson model was found in Refs ${ }^{4}$ and ${ }^{12}$, respectively, thus making a connection between the WCL and free probability. The WCL is typically described by the quantum Boltzmann statistics ${ }^{4}$. In Re ${ }^{13}$ a generalized version of Boltzmann commutation relations, the so called entangled commutation relations, was found in the weak coupling limit for nonlinear interactions and possible applications to photon splitting cascades were discussed.

The investigation of the multitime non-chronologically ordered correlation functions could have a connection with the behavior of fluctuations in certain asymptotic regimes. The latter is described in the review paper by Andries, Benatti, De Cock and Fannes ${ }^{14}$. In that approach the limiting statistics is defined in terms of ground state distribution determined by non trivial pair partitions. The authors conjecture the appearance of exotic statistics in certain asymptotic regimes. The asymptotic fluctuations are the limiting correlation functions of appropriate centered elements and thus the results of the present paper could be applied to study the fluctuations in the low density limit.

In Sec. II the truncated non-chronologically ordered correlation functions are defined and their low density limit is established (Theorem 11). In Sec. III the irreducible diagrams (pair partitions) which contribute to the limiting correlation functions are found (Theorem 2). In Sec. IV the limiting truncated correlation functions are represented as correlation functions of a suitable free white noise. In Sec. V we identify the independent in the limit subalgebras (Theorem 4) and calculate the limiting cumulants which for some elements coincide with the cumulants of the Poisson distribution (Theorem 5). In Sec. VI an explicit representation of the limiting correlation functions and thus of the limiting algebra is constructed for a special case by using suitable quantum white noise operators.

\section{THE CORRELATION FUNCTIONS IN THE LDL}

We begin this section with construction of a general class of non-commutative probability spaces relevant for the investigation of the low density limit. The framework of a *- 
probability space is used. A relation between the objects defined in this section and the model of a test particle interacting with a dilute gas is given in Appendix A.

Definition $1 A$ *-probability space is a pair $(\mathcal{A}, \omega)$, where $\mathcal{A}$ is a unital $*$-algebra over $\mathbb{C}$ and $\omega: \mathcal{A} \rightarrow \mathbb{C}$ is a state, i.e., a linear normalized, $\omega\left(1_{\mathcal{A}}\right)=1$, and strictly positive functional.

Let $\mathcal{H}$ be a Hilbert space with inner product denoted by $\langle\cdot, \cdot\rangle$ (called as one particle Hilbert space), $\left\{S_{t}\right\}_{t \in \mathbb{R}}$ a one parameter unitary group in $\mathcal{H}$ (a one particle free evolution), $\hat{n}$ a bounded positive operator in $\mathcal{H}$ (density operator) such that $\forall t \in \mathbb{R}$, $S_{-t} \hat{n} S_{t}=\hat{n}$, and $B$ a countable set of real numbers.

Let $\Gamma(\mathcal{H})$ be the symmetric Fock space over $\mathcal{H}$. For any trace class self-adjoint operator $T$ acting in $\mathcal{H}$ we denote by $N(T) \equiv \mathrm{d} \Gamma(T)$ its second quantization operator in $\Gamma(\mathcal{H})$ and extend this definition by complex linearity to the set of all trace class operators $\mathcal{T}(\mathcal{H})$. For any $T \in \mathcal{T}(\mathcal{H}), \omega \in B$, and a positive number $\varepsilon>0$ we define the following operator in $\Gamma(\mathcal{H})$ :

$$
N_{T, \omega, \varepsilon}(t):=\frac{\mathrm{e}^{-\mathrm{i} t \omega / \varepsilon}}{\varepsilon} N\left(S_{t / \varepsilon} T S_{-t / \varepsilon}\right)
$$

Let $L(\mathbb{R})=\bigcap_{p \in \mathbb{N}} L^{p}(\mathbb{R})$, where $L^{p}(\mathbb{R})$ is the space of $p$-power intergable functions over $\mathbb{R}$. For any open subset $\Lambda \subseteq \mathbb{R}$ let $L(\Lambda)$ be the set of functions from $L(\mathbb{R})$ with support in $\Lambda$. We denote by $\mathcal{A}_{\Lambda, \varepsilon}$ the $*$-algebra generated by operators $N_{T, \omega, \varepsilon}(\varphi):=\int \mathrm{d} t \varphi(t) N_{T, \omega, \varepsilon}(t)$ with $T \in \mathcal{T}(\mathcal{H}), \omega \in B, \varphi \in S(\Lambda)$ and denote $\mathcal{A}_{\varepsilon}:=\mathcal{A}_{\mathbb{R}, \varepsilon}$.

Let $A^{ \pm}(g), g \in \mathcal{H}$ be the creation and annihilation operators in $\Gamma(\mathcal{H})$ [we denote in the sequel $\left.A^{-}(g) \equiv A(g)\right]$ with the canonical commutation relations $\left[A(f), A^{+}(g)\right]=$ $\langle f, g\rangle$ and let $\mathcal{A}_{\mathrm{CCR}}$ be the algebra of polynomials in $A^{ \pm}(\cdot)$. Any operator $N(T)$ can be represented in terms of the creation and annihilation operators. For example, if $T=$ $|f\rangle\langle g|$, where we use Dirac's notations for elements $f, g \in \mathcal{H}$, then $N(T)=A^{+}(f) A(g)$. An arbitrary operator $N(T)$ can be expressed in terms of $A^{ \pm}$using the fact that any trace class operator $T$ is a limit of finite rank operators. Thus the algebra $\mathcal{A}_{\varepsilon}$ is a subalgebra of $\mathcal{A}_{\mathrm{CCR}}$.

Let $\omega_{\hat{n}}$ be a gaussian gauge-invariant mean-zero state on $\mathcal{A}_{\mathrm{CCR}}$ with the two point correlation function $\omega_{\hat{n}}\left(A^{+}(f) A(g)\right):=\langle g, \hat{n} f\rangle$ (thus $\omega_{\hat{n}}(N(T))=\operatorname{Tr}(\hat{n} T)$ and here we use the assumption for $T$ being trace class). Denoting by the same symbol its restriction to $\mathcal{A}_{\Lambda, \varepsilon}$, we finally have for any $\varepsilon>0$ and for any open subset $\Lambda \subseteq \mathbb{R}$ the $*$-probability space $\left(\mathcal{A}_{\Lambda, \varepsilon}, \omega_{\varepsilon \hat{n}}\right)$.

Remark 1 The condition $\forall t: S_{-t} \hat{n} S_{t}=\hat{n}$ leads to the invariance of the state $\omega_{\hat{n}}$ under the free evolution generated by $S_{t}$.

With the notations above we define the non-chronologically ordered multitime correlation functions as

$$
\begin{aligned}
& W_{\varepsilon, \hat{n}, T_{1}, \omega_{1}, \ldots, T_{n}, \omega_{n}}\left(t_{1}, \ldots, t_{n}\right):=\omega_{\varepsilon \hat{n}}\left(N_{T_{1}, \omega_{1}, \varepsilon}\left(t_{1}\right) \ldots N_{T_{n}, \omega_{n}, \varepsilon}\left(t_{n}\right)\right) \\
& W_{\varepsilon, \hat{n}, T_{1}, \omega_{1}, \ldots, T_{n}, \omega_{n}}\left(\varphi_{1}, \ldots, \varphi_{n}\right):=\omega_{\varepsilon \hat{n}}\left(N_{T_{1}, \omega_{1}, \varepsilon}\left(\varphi_{1}\right) \ldots N_{T_{n}, \omega_{n}, \varepsilon}\left(\varphi_{n}\right)\right)
\end{aligned}
$$


We will use for the correlation functions (2) and (3) also the shorter notations $W_{\varepsilon}\left(t_{1}, \ldots, t_{n}\right)$ and $W_{\varepsilon}\left(\varphi_{1}, \ldots, \varphi_{n}\right)$. The reason for introducing the averaged operators $N_{T, \omega, \varepsilon}(\varphi)$ and the averaged correlation functions (3) is that, as we will show below, the non-averaged operators $N_{T, \omega, \varepsilon}(t)$ and the correlation functions (2) in the limit as $\varepsilon \rightarrow 0$ become singular distributions. Clearly, one has the relation

$$
W_{\varepsilon}\left(\varphi_{1}, \ldots, \varphi_{n}\right)=\int \mathrm{d} t_{1} \ldots \mathrm{d} t_{n} W_{\varepsilon}\left(t_{1}, \ldots, t_{n}\right) \varphi_{1}\left(t_{1}\right) \ldots \varphi_{n}\left(t_{n}\right)
$$

Definition 2 The truncated correlation functions $W_{\varepsilon}^{T}\left(t_{1}, \ldots, t_{n}\right)$ are defined for $n=1$ by $W_{\varepsilon}^{T}\left(t_{1}\right):=W_{\varepsilon}\left(t_{1}\right)$ and for $n>1$ by induction through the relation:

$$
\begin{aligned}
W_{\varepsilon}\left(t_{1}, \ldots, t_{n}\right)= & W_{\varepsilon}^{T}\left(t_{1}, \ldots, t_{n}\right)+\sum_{l=2}^{n} \sum^{\prime} W_{\varepsilon}^{T}\left(t_{i_{1}}, \ldots, t_{i_{k_{1}}}\right) \\
& \times W_{\varepsilon}^{T}\left(t_{i_{k_{1}+1}}, \ldots, t_{i_{k_{2}}}\right) \ldots W_{\varepsilon}^{T}\left(t_{i_{k_{l}}}, \ldots, t_{i_{n}}\right)
\end{aligned}
$$

where $\sum^{\prime}$ is the sum over $i_{1}<i_{2}<\ldots<i_{k_{1}}, i_{k_{1}+1}<\ldots<i_{k_{2}}, \ldots, i_{k_{l}+1}<\ldots<i_{n}$.

The truncated correlation functions are often used in quantum field theory and in quantum kinetic theory 15 . They entirely determine the corresponding non-chronological correlation functions. Thus the investigation of the limit of the non-chronological correlation functions can be reduced to the investigation of the limit of the truncated correlation functions.

We define the "projection" $P_{E}:=(2 \pi)^{-1} \int \mathrm{d} t S_{t} \mathrm{e}^{-\mathrm{i} t E}$ [it has the property $P_{E} P_{E^{\prime}}=$ $\left.\delta\left(E-E^{\prime}\right) P_{E}\right]$ and for any $k=1,2, \ldots, n$ denote $\tilde{\omega}_{k}=\omega_{n}+\ldots+\omega_{k}$. The following theorem states the low density limit of the truncated correlation functions.

Theorem 1 One has the limit in the sense of distributions in variables $t_{1}, \ldots, t_{n}$ :

$$
\begin{aligned}
\lim _{\varepsilon \rightarrow 0} W_{\varepsilon, \hat{n}, T_{1}, \omega_{1}, \ldots, T_{n}, \omega_{n}}^{T}\left(t_{1}, \ldots, t_{n}\right)= & (2 \pi)^{n-1} \delta\left(t_{2}-t_{1}\right) \ldots \delta\left(t_{n}-t_{n-1}\right) \\
& \times \delta_{\tilde{\omega}_{1}, 0} \int \mathrm{d} E \operatorname{Tr}\left[\hat{n} P_{E+\tilde{\omega}_{1}} T_{1} P_{E+\tilde{\omega}_{2}} T_{2} \ldots P_{E+\tilde{\omega}_{n}} T_{n}\right]
\end{aligned}
$$

where $\operatorname{Tr}$ denotes trace and $\delta_{\tilde{\omega}_{1}, 0}$ is the Kronecker delta symbol.

The theorem is a corollary of Theorem 2 from Section 3.

\section{THE NON-TRIVIAL DIAGRAMS}

In the present section we investigate the low density limit of the non-chronologically ordered correlation functions for the particular case of operators of the form $T_{l}=\left|f_{l}\right\rangle\left\langle g_{l}\right|$ and find the diagrams which are non-trivial in the low density limit.

In order to simplify the notations we will use the following energy representation for the creation and annihilation operators:

$$
A_{l}^{+}:=\frac{\mathrm{e}^{\mathrm{i} t_{l} E_{l} / \varepsilon}}{\sqrt{\varepsilon}} A^{+}\left(P_{E_{l}} f_{l}\right) ; \quad A_{l}:=\frac{1}{\sqrt{\varepsilon}} A\left(S_{t_{l} / \varepsilon} g_{l}\right)
$$


(a slightly different version of the energy representation was introduced in ${ }^{7}$ ). One has $N_{T_{l}, \omega_{l}, \varepsilon}\left(t_{l}\right)=\mathrm{e}^{-\mathrm{i} t_{l} \omega_{l} / \varepsilon} \int \mathrm{d} E_{l} A_{l}^{+} A_{l}$. Notice that the operator $A_{l}^{+}$is not the adjoint of $A_{l}$. The symbols $A_{l}, A_{l}^{+}$are used only to simplify the notations below.

A multitime correlation function can be expressed using Gaussianity of the state $\omega_{\hat{n}}$ and the energy representation for the creation and annihilation operators as

$$
\begin{aligned}
W_{\varepsilon, \hat{n}, T_{1}, \omega_{1}, \ldots, T_{n}, \omega_{n}}\left(t_{1}, \ldots, t_{n}\right)= & \exp \left(-\mathrm{i} \sum_{l=1}^{n} \omega_{l} t_{l} / \varepsilon\right) \sum^{\prime} \int \mathrm{d} E_{1} \ldots \mathrm{d} E_{n} \omega_{\varepsilon \hat{n}}\left(A_{i_{1}}^{+} A_{j_{1}}\right) \ldots \\
& \times \omega_{\varepsilon \hat{n}}\left(A_{i_{k}}^{+} A_{j_{k}}\right) \omega_{\varepsilon \hat{n}}\left(A_{j_{k+1}} A_{i_{k+1}}^{+}\right) \ldots \omega_{\varepsilon \hat{n}}\left(A_{j_{n}} A_{i_{n}}^{+}\right)
\end{aligned}
$$

where $\sum^{\prime}$ is the sum over $k=1, \ldots, n, 1=i_{1}<i_{2}<\ldots<i_{k}, j_{k+1}<\ldots<j_{n}, i_{l} \leq j_{l}$ for $l=1, \ldots, k$ and $j_{l}<i_{l}$ for $l=k+1, \ldots, n$. The sum contains terms of the form

$$
\omega_{\varepsilon \hat{n}}\left(A_{i_{1}}^{+} A_{j_{1}}\right) \ldots \omega_{\varepsilon \hat{n}}\left(A_{i_{k}}^{+} A_{j_{k}}\right) \omega_{\varepsilon \hat{n}}\left(A_{j_{k+1}} A_{i_{k+1}}^{+}\right) \ldots \omega_{\varepsilon \hat{n}}\left(A_{j_{n}} A_{i_{n}}^{+}\right)
$$

To each such term we associate a diagram by pairing in the string $A_{1}^{+} A_{1} A_{2}^{+} A_{2} \ldots A_{n}^{+} A_{n}$ the operators $A_{i_{l}}^{+}$and $A_{j_{l}}$ for $l=1,2, \ldots n$.

Definition 3 We say that the expression (6) corresponds to a reducible diagram if there exists a nonempty subset $I \subset\{1, \ldots, n\}$ (strict inclusion) such that $i_{l} \in I \Leftrightarrow j_{l} \in I$. Otherwise we say that the expression (6) corresponds to an irreducible diagram.

An important property of the truncated correlation functions (Def. 22) is that they keep only all irreducible diagrams. The following are the examples of irreducible (first) and reducible (second) diagrams for $n=2$ :

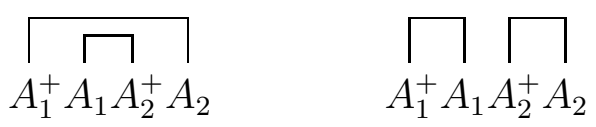

Given an reducible diagram, one can represent the set $\{1, \ldots n\}$ as a union of several disjoint subsets $I_{1}, \ldots, I_{l}$ such that the diagram contains only pairings between operators with indices from the same subsets. In this sense a general reducible diagram can be represented as a union of mutually disjoint irreducible diagrams. Examples of the truncated correlation functions, the corresponding irreducible diagrams, and their limits as $\varepsilon \rightarrow 0$ for $n=1,2,3$ are given below.

Example $1 n=1$. The invariance of the state under the free evolution leads to the identity $W_{\varepsilon}^{T}(t) \equiv W_{\varepsilon}(t) \equiv W_{\varepsilon}(0)=\left\langle g_{1}, \hat{n} f_{1}\right\rangle$.

Example $2 n=2$. One has

$$
\begin{aligned}
W_{\varepsilon}^{T}\left(t_{1}, t_{2}\right) & =W_{\varepsilon}\left(t_{1}, t_{2}\right)-W_{\varepsilon}\left(t_{1}\right) W_{\varepsilon}\left(t_{2}\right)=\int \mathrm{d} E_{1} \mathrm{~d} E_{2} \omega_{\varepsilon \hat{n}}\left(A_{1}^{+} A_{2}\right) \omega_{\varepsilon \hat{n}}\left(A_{1} A_{2}^{+}\right) \\
& =\int \mathrm{d} E_{1} \mathrm{~d} E_{2} \frac{\mathrm{e}^{\mathrm{i}\left(t_{2}-t_{1}\right)\left(E_{2}-E_{1}\right) / \varepsilon}}{\varepsilon}\left\langle g_{2}, P_{E_{1}} \hat{n} f_{1}\right\rangle\left\langle g_{1},(1+\varepsilon \hat{n}) P_{E_{2}} f_{2}\right\rangle(8)
\end{aligned}
$$

This expression corresponds to the first (irreducible) diagram in (7) which is non-zero in the limit. Application of Lemma 1 (see Appendix B) to the r.h.s. of (8) gives

$$
\lim _{\varepsilon \rightarrow 0} W_{\varepsilon}^{T}\left(t_{1}, t_{2}\right)=2 \pi \delta\left(t_{2}-t_{1}\right) \int \mathrm{d} E\left\langle g_{2}, P_{E} \hat{n} f_{1}\right\rangle\left\langle g_{1}, P_{E} f_{2}\right\rangle
$$


Example $3 n=3$. One has

$$
\begin{aligned}
W_{\varepsilon}^{T}\left(t_{1}, t_{2}, t_{3}\right)= & \int \mathrm{d} E_{1} \mathrm{~d} E_{2} \mathrm{~d} E_{3}\left[\omega_{\varepsilon \hat{n}}\left(A_{1}^{+} A_{3}\right) \omega_{\varepsilon \hat{n}}\left(A_{1} A_{2}^{+}\right) \omega_{\varepsilon \hat{n}}\left(A_{2} A_{3}^{+}\right)\right. \\
& \left.+\omega_{\varepsilon \hat{n}}\left(A_{1}^{+} A_{2}\right) \omega_{\varepsilon \hat{n}}\left(A_{1} A_{3}^{+}\right) \omega_{\varepsilon \hat{n}}\left(A_{2}^{+} A_{3}\right)\right]
\end{aligned}
$$

This expression corresponds to the sum of the two irreducible diagrams:

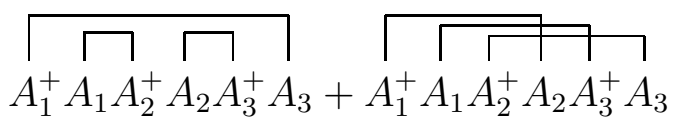

In this case only the first diagram is non-zero in the limit and Lemma 1 gives $\lim _{\varepsilon \rightarrow 0} W_{\varepsilon}^{T}\left(t_{1}, t_{2}, t_{3}\right)=(2 \pi)^{2} \delta\left(t_{3}-t_{2}\right) \delta\left(t_{2}-t_{1}\right) \int \mathrm{d} E\left\langle g_{3}, P_{E} \hat{n} f_{1}\right\rangle\left\langle g_{1}, P_{E} f_{2}\right\rangle\left\langle g_{2}, P_{E} f_{3}\right\rangle$

The case of arbitrary $n$ is described by the following theorem.

Theorem 2 Let $T_{l}=\left|f_{l}\right\rangle\left\langle g_{l}\right|$, where $f_{l}, g_{l} \in \mathcal{H}$ for $l=1,2, \ldots, n$. One has the limit in the sense of distributions in variables $t_{1}, \ldots, t_{n}$ :

$$
\begin{aligned}
& \lim _{\varepsilon \rightarrow 0} W_{\varepsilon, \hat{n}, T_{1}, \omega_{1}, \ldots, T_{n}, \omega_{n}}^{T}\left(t_{1}, \ldots, t_{n}\right)=(2 \pi)^{n-1} \delta\left(t_{2}-t_{1}\right) \ldots \delta\left(t_{n}-t_{n-1}\right) \\
& \times \delta_{\tilde{\omega}_{1}, 0} \int \mathrm{d} E\left\langle g_{n}, P_{E} \hat{n} f_{1}\right\rangle\left\langle g_{1}, P_{E+\tilde{\omega}_{2}} f_{2}\right\rangle \ldots\left\langle g_{n-1}, P_{E+\tilde{\omega}_{n}} f_{n}\right\rangle
\end{aligned}
$$

For each $n$ only the following irreducible diagram is non-zero as $\varepsilon \rightarrow 0$ :

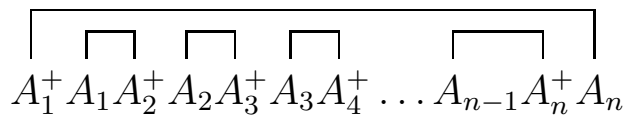

Proof. Case (a): $\omega_{1}=\omega_{2}=\ldots=\omega_{n}=0$. Using the correlation functions

$$
\begin{aligned}
& \omega_{\varepsilon \hat{n}}\left(A_{i_{\alpha}}^{+} A_{j_{\alpha}}\right)=\mathrm{e}^{\mathrm{i}\left(t_{i_{\alpha}}-t_{j_{\alpha}}\right) E_{i_{\alpha}} / \varepsilon}\left\langle g_{j_{\alpha}}, \hat{n} f_{i_{\alpha}}\right\rangle \\
& \omega_{\varepsilon \hat{n}}\left(A_{j_{\beta}} A_{i_{\beta}}^{+}\right)=\frac{\mathrm{e}^{\mathrm{i}\left(t_{i_{\beta}}-t_{j_{\beta}}\right) E_{i_{\beta}} / \varepsilon}}{\varepsilon}\left\langle g_{j_{\beta}},(1+\varepsilon \hat{n}) f_{i_{\beta}}\right\rangle
\end{aligned}
$$

one can write (6) as

$$
\frac{1}{\varepsilon^{n}} \exp \left\{\mathrm{i}\left[\left(t_{1}-t_{j_{1}}\right) E_{1}+\ldots+\left(t_{i_{n}}-t_{j_{n}}\right) E_{i_{n}}\right] / \varepsilon\right\}\left(\varepsilon^{k} F(E)+O\left(\varepsilon^{k+1}\right)\right)
$$

where

$$
F(E)=\prod_{l=1}^{k}\left\langle g_{j_{l}}, P_{E_{l}} \hat{n} f_{i_{l}}\right\rangle \prod_{l=k+1}^{n}\left\langle g_{j_{l}}, P_{E_{i_{l}}} f_{i_{l}}\right\rangle
$$

Define the permutations $p_{i}$ and $p_{j}$ of the set $(1, \ldots, n)$ by $p_{i}(l)=i_{l}$ and $p_{j}(l)=j_{l}$ for $l=1, \ldots, n$ and let $p_{\alpha}=p_{i} p_{j}^{-1}$. Consider the expression in the square brackets in the exponent in (11). The term proportional to $t_{l}$ in this expression has the form $t_{l}\left(E_{l}-E_{\alpha_{l}}\right)$, where $\alpha_{l}=p_{\alpha}(l)$. Thus (11) can be written as

$$
\frac{1}{\varepsilon^{n}} \exp \left\{\mathrm{i}\left[t_{n}\left(E_{n}-E_{\alpha_{n}}\right)+\ldots+t_{1}\left(E_{1}-E_{\alpha_{1}}\right)\right] / \varepsilon\right\}\left(\varepsilon^{k} F(E)+O\left(\varepsilon^{k+1}\right)\right)
$$

and with the notations $\Omega_{l}(E)=E_{n}+\ldots+E_{l}-E_{\alpha_{n}}-\ldots-E_{\alpha_{l}}$ for $l=2, \ldots, n$ as

$$
\frac{\mathrm{e}^{\mathrm{i}\left(t_{n}-t_{n-1}\right) \Omega_{n}(E) / \varepsilon}}{\varepsilon} \ldots \frac{\mathrm{e}^{\mathrm{i}\left(t_{2}-t_{1}\right) \Omega_{2}(E) / \varepsilon}}{\varepsilon}\left(\varepsilon^{k-1} F(E)+O\left(\varepsilon^{k}\right)\right)
$$


If the expression (6) corresponds to an irreducible diagram then the functions $\Omega_{l}(E)$ are linearly independent and, since they are linear in their arguments, the convolution $\delta\left(\Omega_{2}(E)\right) \ldots \delta\left(\Omega_{n}(E)\right)$ is well defined.

In the case $k>1$, since for any $l=2, \ldots, n$ (see Lemma 1):

$$
\lim _{\varepsilon \rightarrow 0} \frac{\mathrm{e}^{\mathrm{i}\left(t_{l}-t_{l-1}\right) \Omega_{l}(E) / \varepsilon}}{\varepsilon}=2 \pi \delta\left(t_{l}-t_{l-1}\right) \delta\left(\Omega_{l}(E)\right)
$$

and $k-1>0$, the limit of (12) equals to zero.

In the case $k=1$ the expression (6) corresponds to the diagram (10) and one has

$$
\begin{aligned}
& \omega_{\varepsilon \hat{n}}\left(A_{1}^{+} A_{n}\right) \omega_{\varepsilon \hat{n}}\left(A_{1} A_{2}^{+}\right) \ldots \omega_{\varepsilon \hat{n}}\left(A_{n-1} A_{n}^{+}\right) \\
\varepsilon & \frac{\mathrm{e}^{\mathrm{i}\left(t_{n}-t_{n-1}\right) \Omega_{n}(E) / \varepsilon}}{\varepsilon} \ldots \frac{\mathrm{e}^{\mathrm{i}\left(t_{2}-t_{1}\right) \Omega_{2}(E) / \varepsilon}}{\varepsilon}(F(E)+O(\varepsilon))
\end{aligned}
$$

where $\Omega_{l}(E)=E_{l}-E_{1}$. Using (13) one finds that the limit of the r.h.s. of (14) is

$$
\begin{aligned}
& (2 \pi)^{n-1} \delta\left(t_{2}-t_{1}\right) \ldots \delta\left(t_{n}-t_{n-1}\right) \delta\left(E_{2}-E_{1}\right) \ldots \delta\left(E_{n}-E_{1}\right) \\
& \times\left\langle g_{n}, P_{E_{1}} \hat{n} f_{1}\right\rangle\left\langle g_{1}, P_{E_{2}} f_{2}\right\rangle \ldots\left\langle g_{n-1}, P_{E_{n}} f_{n}\right\rangle
\end{aligned}
$$

Integration over $E_{1} \ldots E_{n}$ gives the equality (9) in the case (a).

Case (b): arbitrary $\omega_{1}, \ldots, \omega_{n}$. In this case the expression (14) in the decomposition (5) is multiplied by the factor $\exp \left(-\mathrm{i} \sum_{l} \omega_{l} t_{l} / \varepsilon\right)$. The product can be written as

$$
\frac{\mathrm{e}^{\mathrm{i}\left(t_{n}-t_{n-1}\right)\left(\Omega_{n}(E)-\tilde{\omega}_{n}\right) / \varepsilon}}{\varepsilon} \ldots \frac{\mathrm{e}^{\mathrm{i}\left(t_{2}-t_{1}\right)\left(\Omega_{2}(E)-\tilde{\omega}_{2}\right) / \varepsilon}}{\varepsilon} \mathrm{e}^{-\mathrm{i} t_{1} \tilde{\omega}_{1} / \varepsilon}(F(E)+O(\varepsilon))
$$

If $\tilde{\omega}_{1}=0$ then the statement of the theorem follows by the same arguments as in the case $(\mathrm{a})$. If $\tilde{\omega}_{1} \neq 0$ then the limit of this term equals to zero by Riemann-Lebesgue lemma due to the presence of the rapidly oscillating factor $\exp \left(-\mathrm{i} t_{1} \tilde{\omega}_{1} / \varepsilon\right)$.

\section{THE FREE WHITE NOISE NUMBER OPERATORS}

In the present section we show that the limiting truncated correlation functions coincide with the complete (i.e., non-truncated) correlation functions of the free white noise number operators.

Definition 4 Free white noise operators $N_{T}(t)$ are the operators satisfying the multiplication rule

$$
N_{T}(t) N_{T^{\prime}}\left(t^{\prime}\right)=\delta\left(t-t^{\prime}\right) N_{T * T^{\prime}}(t)
$$

where the *-product of any two operators $T$ and $T^{\prime}$ is defined by $T * T^{\prime}:=$ $2 \pi \int \mathrm{d} E P_{E} T P_{E} T^{\prime}$.

Remark 2 We call the operators $N_{T}(t)$ as free (or Boltzmann) number operators since they can be constructed using the creation and annihilation operators $B_{f}^{ \pm}(t)$ satisfying the free relations $B_{f}^{-}(t) B_{g}^{+}\left(t^{\prime}\right)=2 \pi \delta\left(t-t^{\prime}\right)\langle f, g\rangle$. In fact, define $N_{|f\rangle\langle g|}(t):=$ $\int \mathrm{d} E B_{P_{E}}^{+}(t) B_{P_{E} g}^{-}(t)$ and extend this definition by linearity to any $T$. Then such defined operators satisfy the relation (15). 
Let $\mathcal{A}$ be the algebra generated by the free white noise operators $N_{T}(t)$ and let $\phi_{\hat{n}}$ be the state on $\mathcal{A}$ characterized by $\phi_{\hat{n}}\left(N_{T}(t)\right)=\operatorname{Tr}(\hat{n} T)$.

Theorem 3 One has the equality

$$
\lim _{\varepsilon \rightarrow 0} W_{\varepsilon, \hat{n}, T_{1}, 0, \ldots, T_{n}, 0}^{\mathrm{T}}\left(t_{1}, \ldots, t_{n}\right)=\phi_{\hat{n}}\left(N_{T_{1}}\left(t_{1}\right) \ldots N_{T_{n}}\left(t_{n}\right)\right)
$$

Proof. By direct calculations using the Eq. (4) and the relation (15).

The existence of the representation of the limiting truncated correlation functions by the free white noise number operators is related to the fact that only a sub-class of the non-crossing irreducible diagrams survives in the low density limit. We emphasize however, that the 1.h.s. of Eq. (16) is the limit of a truncated correlation function whereas the r.h.s. contains the complete correlation function.

\section{INDEPENDENCE AND THE GENERALIZED POISSON STATISTICS IN THE LDL}

The fact that the limiting truncated correlation functions are the distributions in variables $t_{1}, \ldots, t_{n}$ with support at $t_{1}=\ldots=t_{n}$ leads to the appearance of independent subalgebras in the low density limit. In the beginning of this section we remind the basic notions of independent subalgebras and of cumulants. Then we find the asymptotically independent subalgebras of $\mathcal{A}_{\varepsilon}$ and discuss the limiting statistics. We show that the cumulants and the moments of certain elements in the algebra $\mathcal{A}_{\varepsilon}$ in the low density limit coincide with the cumulants and the moments of the Poisson distribution.

Definition 5 Let $(\mathcal{A}, \omega)$ be a *-probability space. A family of unital $*$-subalgebras $\left\{\mathcal{A}_{i}\right\}_{i \in I}, \mathcal{A}_{i} \subset \mathcal{A}$, is called independent if $\omega\left(a_{1} \ldots a_{n}\right)=0$ whenever $a_{l} \in \mathcal{A}_{i_{l}}$, $\omega\left(a_{l}\right)=0$, and $k \neq l$ implies $i_{k} \neq i_{l}$.

Definition 6 Let $(\mathcal{A}, \omega)$ be a *-probability space. Cumulants of the space $(\mathcal{A}, \omega)$ are the multilinear functionals $\kappa_{n}: \mathcal{A}^{n} \rightarrow \mathbb{C}, n \geq 1$, uniquely determined by $\kappa_{1}(a):=$ $\omega(a), a \in \mathcal{A}$, and for $n>1$ by induction through the relation:

$$
\omega\left(a_{1} \ldots a_{n}\right)=\sum_{\pi, \pi=:\left\{A_{1}, \ldots, A_{k}\right\}} \kappa_{\left|A_{j}\right|}\left(\left(a_{1}, \ldots, a_{n}\right) \mid A_{j}\right)
$$

where the sum is over all partitions $\pi$ of the set $\{1, \ldots, n\}$ and $"\left(a_{1}, \ldots, a_{n}\right) \mid A "$ designates the set of $a_{i}$ with $i \in A$.

Remark 3 The cumulants $\kappa_{n}^{(\varepsilon)}$ for a $*$-probability space $\left(\mathcal{A}_{\varepsilon}, \omega_{\varepsilon \hat{n}}\right)$ are directly related to the truncated correlation functions. Namely, if $a_{1}=N_{T_{1}, \omega_{1}, \varepsilon}\left(\varphi_{1}\right), \ldots, a_{n}=N_{T_{n}, \omega_{n}, \varepsilon}\left(\varphi_{n}\right)$, then $\kappa_{n}^{(\varepsilon)}\left(a_{1}, \ldots, a_{n}\right)=W_{\varepsilon, \hat{n}, T_{1}, \omega_{1}, \ldots, T_{n}, \omega_{n}}^{T}\left(\varphi_{1}, \ldots, \varphi_{n}\right)$.

For the analysis of independence in the low density limit we introduce the notion of asymptotically independent subalgebras for a $*$-probability space $\left(\mathcal{A}_{\varepsilon}, \omega_{\varepsilon \hat{n}}\right)$. 
Definition 7 Let $\left(\mathcal{A}_{\varepsilon}, \omega_{\varepsilon \hat{n}}\right)$ be a *-probability space for the $L D L$. We say that a family of subalgebras $\mathcal{A}_{1, \varepsilon}, \ldots, \mathcal{A}_{l, \varepsilon}$ of $\mathcal{A}_{\varepsilon}$ is asymptotically independent if

$$
\lim _{\varepsilon \rightarrow 0} \omega_{\varepsilon \hat{n}}\left(a_{1}, \ldots, a_{n}\right)=0
$$

whenever $a_{l} \in \mathcal{A}_{i_{l}, \varepsilon}, \omega_{\varepsilon \hat{n}}\left(a_{l}\right)=0$, and $k \neq l$ implies $i_{k} \neq i_{l}$.

The next theorem identifies asymptotically independent subalgebras of $\mathcal{A}_{\varepsilon}$.

Theorem 4 Let $\Lambda_{1}, \ldots, \Lambda_{l}$ be a family of disjoint open subsets in $\mathbb{R}$. Then the family of subalgebras $\mathcal{A}_{\Lambda_{1}, \varepsilon}, \ldots, \mathcal{A}_{\Lambda_{l}, \varepsilon}$ is asymptotically independent.

The proof follows from the fact that the truncated correlation functions become in the limit as $\varepsilon \rightarrow 0$ distributions in variables $t_{1}, \ldots, t_{n}$ with support at $t_{1}=t_{2}=\ldots=t_{n}$.

Now let us analyze the statistics which appears in the low density limit. From Theorem 1 and the relation between the cumulants and the truncated correlation functions it follows that $l$-th cumulant for the element $a=N_{T, \omega, \varepsilon}(\varphi)$ in the limit has the form

$\kappa_{l}(a, \ldots, a)=\lim _{\varepsilon \rightarrow 0} W_{\varepsilon, \hat{n}, T, \omega, \ldots, T, \omega}^{T}(\varphi, \ldots, \varphi)=\frac{1}{2 \pi} \delta_{\omega, 0} \int \mathrm{d} t \mathrm{~d} E \operatorname{Tr} \hat{n}\left[2 \pi \varphi(t) P_{E} T\right]^{l}$

We specify the further consideration to the case $\mathcal{H}=L^{2}\left(\mathbb{R}^{3}\right)$. Consider $\hat{n}=1$ and $S_{t}=\mathrm{e}^{\mathrm{i} t H_{1}}$ where $H_{1}$ is the multiplication operator by the function $\omega(\mathbf{k})=$ $|\mathbf{k}|^{2}, \mathbf{k} \in \mathbb{R}^{3}$. Let $T_{\lambda}$ be an integral operator in $\mathcal{H}$ with the kernel $T_{\lambda}\left(\mathbf{k}, \mathbf{k}^{\prime}\right)=$ $\left(2 \pi \sqrt{|\mathbf{k}|\left|\mathbf{k}^{\prime}\right|}\right)^{-1} \chi_{[0, \sqrt{\lambda}]}(|\mathbf{k}|) \chi_{[0, \sqrt{\lambda}]}\left(\left|\mathbf{k}^{\prime}\right|\right)$, where $\lambda$ is a positive number and $\chi_{[0, \sqrt{\lambda}]}$ is the characteristic function of the interval $[0, \sqrt{\lambda}]$. Let $\varphi_{0}(t)=(2 \pi)^{-1} \chi_{[0,2 \pi]}(t)$.

Theorem 5 Let $a_{\lambda}=N_{T_{\lambda}, \omega, \varepsilon}\left(\varphi_{0}\right)$, where $T_{\lambda}$ and $\varphi_{0}$ are defined as above. Then for any $l \in \mathbb{N}$ one has

$$
\kappa_{l}\left(a_{\lambda}, \ldots, a_{\lambda}\right)=\lambda \delta_{\omega, 0}
$$

or equivalently, the cumulants of the element $a_{\lambda}$ with $\omega=0$ coincide in the low density limit with the cumulants of the Poisson distribution with expectation equal to $\lambda$.

Proof. The proof of the theorem is based on the direct calculation of the cumulants using Eq. (17). One has

$$
\frac{1}{2 \pi} \int \mathrm{d} t\left[2 \pi \varphi_{0}(t)\right]^{l}=1
$$

One also has

$$
\begin{aligned}
\int \mathrm{d} E \operatorname{Tr} \hat{n}\left[P_{E} T_{\lambda}\right]^{l}= & \int \mathrm{d} E \int \mathrm{d} \mathbf{k}_{1} \ldots \mathrm{d} \mathbf{k}_{l} \delta\left(\left|\mathbf{k}_{1}\right|^{2}-E\right) T_{\lambda}\left(\mathbf{k}_{1}, \mathbf{k}_{2}\right) \\
& \times \delta\left(\left|\mathbf{k}_{2}\right|^{2}-E\right) T_{\lambda}\left(\mathbf{k}_{2}, \mathbf{k}_{3}\right) \ldots \delta\left(\left|\mathbf{k}_{l}\right|^{2}-E\right) T_{\lambda}\left(\mathbf{k}_{l}, \mathbf{k}_{1}\right) \\
= & \int \mathrm{d} E\left[T_{\lambda}(\sqrt{E}, \sqrt{E}) \int \mathrm{d} \mathbf{k} \delta\left(|\mathbf{k}|^{2}-E\right)\right]^{l}=\int \mathrm{d} E \chi_{[0, \sqrt{\lambda}]}(\sqrt{E})=\lambda .
\end{aligned}
$$

Thus the r.h.s. of Eq. (17) equals to one. This proves the theorem. 
Moments of the element $a_{\lambda}$ with $\omega=0$ in the low density limit are equal to the sum over all partitions of the limiting cumulants and given by Touchard polynomials:

$$
\lim _{\varepsilon \rightarrow 0} \omega_{\varepsilon \hat{n}}\left(a_{\lambda}^{n}\right)=\sum_{k=1}^{n} S(n, k) \lambda^{k}
$$

where $S(n, k)$ is a Stirling number of the second kind, i.e., the number of partitions of a set of size $n$ into $k$ disjoint non-empty subsets. The limiting moments coincide with the moments of the Poisson distribution with expectation equal to $\lambda$. For $a_{1}$ one has

$$
\lim _{\varepsilon \rightarrow 0} \omega_{\varepsilon \hat{n}}\left(a_{1}^{n}\right)=B_{n}
$$

where $B_{n}$ is the $n$-th Bell number, i.e., the number of partitions of a set of size $n$. The Bell numbers are the moments of the Poisson distribution with expectation equal to 1.

\section{AN OPERATOR REPRESENTATION OF THE LIMITING CORRELATION FUNCTIONS}

In the present section we explicitly realize the limiting correlation functions as correlation functions of certain operators acting in a suitable Hilbert space. Presence of delta functions in the limiting correlation functions suggests that they can be represented as correlation functions of certain white noise operators. Here such a representation is constructed in the special case using the results of ${ }^{7}$.

Let $g_{0}, g_{1} \in \mathcal{H}$ satisfy the condition $\left\langle g_{0}, S_{t} g_{1}\right\rangle=0$ for any $t \in \mathbb{R}$. Define for $n, m=0,1$ the Hilbert space $\mathcal{K}_{n m}:=L^{2}\left(\operatorname{Spec} H_{1}, \mathrm{~d} \mu_{n m}\right)$, where Spec $H_{1} \subset \mathbb{R}$ is the spectrum of $H_{1}$ and $\mathrm{d} \mu_{n m}:=\left\langle g_{n}, P_{E} g_{n}\right\rangle\left\langle g_{m}, P_{E} \hat{n} g_{m}\right\rangle \mathrm{d} E$. Let $\mathcal{K}:=\bigoplus_{n, m=0,1} \mathcal{K}_{n m}$ and let $\mathcal{H}_{W N}:=\Gamma\left(L^{2}(\mathbb{R}, \mathcal{K})\right)$ be the symmetric Fock space over the Hilbert space of square integrable $\mathcal{K}$-valued functions on $\mathbb{R}$ (abbreviation $W N$ here stands for White Noise). Using the natural decomposition $\mathcal{H}_{W N}=\bigotimes_{n, m=0,1} \Gamma\left(L^{2}\left(\mathbb{R}, \mathcal{K}_{n m}\right)\right)$ one can define the creation and annihilation operator valued distributions $B_{m, n}^{ \pm}(E, t)$ acting in $\mathcal{H}_{W N}$ and satisfying the canonical commutation relations:

$\left[B_{m, n}^{-}(E, t), B_{m^{\prime}, n^{\prime}}^{+}\left(E^{\prime}, t^{\prime}\right)\right]=2 \pi \delta\left(t^{\prime}-t\right) \delta\left(E^{\prime}-E\right)\left\langle g_{m}, P_{E} g_{m^{\prime}}\right\rangle\left\langle g_{n^{\prime}}, P_{E} \hat{n} g_{n}\right\rangle$

The operator valued distributions $B_{m, n}^{ \pm}(E, t)$ are called time-energy quantum white

noise due to the presence of $\delta\left(t^{\prime}-t\right) \delta\left(E-E^{\prime}\right)$ in (18). Let define the number operators

$$
\tilde{N}_{m, n}(E, t):=\sum_{n^{\prime}=0,1} \frac{1}{\left\langle g_{n^{\prime}}, P_{E} \hat{n} g_{n^{\prime}}\right\rangle} B_{m, n^{\prime}}^{+}(E, t) B_{n, n^{\prime}}^{-}(E, t)
$$

and denote $N_{g_{m}, g_{n}}(t):=\int \mathrm{d} E\left[\tilde{N}_{m, n}(E, t)+B_{n, m}^{-}(E, t)+B_{m, n}^{+}(E, t)\right]$. Let $\Omega \in \mathcal{H}_{W N}$ be the vacuum vector.

Theorem 6 Let $T_{1}=\left|g_{m_{1}}\right\rangle\left\langle g_{n_{1}}\left|, \ldots, T_{k}=\right| g_{m_{k}}\right\rangle\left\langle g_{n_{k}}\right|$, where $m_{1}, n_{1}, \ldots, m_{k}, n_{k} \in$ $\{0,1\}$. One has the equality

$$
\lim _{\varepsilon \rightarrow 0} W_{\varepsilon, \hat{n}, T_{1}, 0, \ldots, T_{k}, 0}\left(t_{1}, \ldots, t_{k}\right)=\left\langle\Omega, N_{g_{m_{1}}, g_{n_{1}}}\left(t_{1}\right) \ldots N_{g_{m_{k}}, g_{n_{k}}}\left(t_{k}\right) \Omega\right\rangle
$$


Proof. r.h.s. of (19) has the form

$$
\begin{aligned}
& \left\langle\Omega, N_{g_{m_{1}}, g_{n_{1}}}\left(t_{1}\right) \ldots N_{g_{m_{k}}, g_{n_{k}}}\left(t_{k}\right) \Omega\right\rangle=\int \mathrm{d} E_{1} \ldots \mathrm{d} E_{k}\left\langle\Omega,\left[\tilde{N}_{m_{1}, n_{1}}\left(E_{1}, t_{1}\right)+B_{n_{1}, m_{1}}^{-}\left(E_{1}, t_{1}\right)\right.\right. \\
& \left.\left.+B_{m_{1}, n_{1}}^{+}\left(E_{1}, t_{1}\right)\right] \ldots\left[\tilde{N}_{m_{k}, n_{k}}\left(E_{k}, t_{k}\right)+B_{n_{k}, m_{k}}^{-}\left(E_{k}, t_{k}\right)+B_{m_{k}, n_{k}}^{+}\left(E_{k}, t_{k}\right)\right] \Omega\right\rangle
\end{aligned}
$$

Let us denote $\tilde{N}_{m, n}(t):=\int \mathrm{d} E \tilde{N}_{m, n}(E, t)$. The truncated correlation function corresponds to the term

$$
\int \mathrm{d} E \mathrm{~d} E^{\prime}\left\langle\Omega, B_{n_{1}, m_{1}}^{-}\left(E, t_{1}\right) \tilde{N}_{m_{2}, n_{2}}\left(t_{2}\right) \tilde{N}_{m_{3}, n_{3}}\left(t_{3}\right) \ldots \tilde{N}_{m_{k-1}, n_{k-1}}\left(t_{k-1}\right) B_{m_{k}, n_{k}}^{+}\left(E^{\prime}, t_{k}\right) \Omega\right\rangle
$$

Notice that $\tilde{N}_{m, n}(t) \Omega=0$. Therefore (20) equals to

$\int \mathrm{d} E \mathrm{~d} E^{\prime}\left\langle\Omega,\left[\ldots\left[\left[B_{n_{1}, m_{1}}^{-}\left(E, t_{1}\right), \tilde{N}_{m_{2}, n_{2}}\left(t_{2}\right)\right], \tilde{N}_{m_{3}, n_{3}}\left(t_{3}\right)\right] \ldots \tilde{N}_{m_{k-1}, n_{k-1}}\left(t_{k-1}\right)\right] B_{m_{k}, n_{k}}^{+}\left(E^{\prime}, t_{k}\right) \Omega\right\rangle$

The commutators can be calculated by induction using the canonical commutation relations (18). The result is

$$
\begin{aligned}
(2 \pi)^{k-2} \delta\left(t_{2}-t_{1}\right) \ldots \delta\left(t_{k-1}-t_{k-2}\right) \int \mathrm{d} E \mathrm{~d} E^{\prime}\left\langle g_{n_{1}}, P_{E} g_{m_{2}}\right\rangle \ldots\left\langle g_{n_{k-2}}, P_{E} g_{m_{k-1}}\right\rangle \\
\times\left\langle\Omega, B_{n_{k-1}, m_{1}}^{-}\left(E, t_{k-1}\right) B_{m_{k}, n_{k}}^{+}\left(E^{\prime}, t_{k}\right) \Omega\right\rangle
\end{aligned}
$$

The last two-point correlation function can be calculated using the commutation relations (18). This gives for (21) the expression

$$
(2 \pi)^{k-1} \delta\left(t_{2}-t_{1}\right) \ldots \delta\left(t_{k}-t_{k-1}\right) \int \mathrm{d} E\left\langle g_{n_{1}}, P_{E} g_{m_{2}}\right\rangle \ldots\left\langle g_{n_{k-1}}, P_{E} g_{m_{1}}\right\rangle\left\langle g_{n_{k}}, P_{E} \hat{n} g_{m_{1}}\right\rangle
$$

which coincides with the r.h.s. of (9) in the case $\omega_{1}=\ldots=\omega_{k}=0$.

Remark 4 The limiting correlation functions could be represented as expectations of certain quantum white noise operators in the general case if one could construct a Hilbert space $\mathcal{H}_{W N}$, a vector $\Omega \in \mathcal{H}_{W N}$, and operator valued distributions $B_{f, g}^{ \pm}(E, t)$ and $\tilde{N}_{f, g}(E, t)$ in $\mathcal{H}_{W N}$ with the property $B_{f, g}^{-}(E, t) \Omega=\tilde{N}_{f, g}(E, t) \Omega=0$ and satisfying the commutation relations

$$
\begin{aligned}
{\left[B_{f, g}^{-}(E, t), B_{f^{\prime}, g^{\prime}}^{+}\left(E^{\prime}, t^{\prime}\right)\right]=} & 2 \pi \delta\left(t^{\prime}-t\right) \delta\left(E^{\prime}-E\right)\left\langle f, P_{E} f^{\prime}\right\rangle\left\langle g^{\prime}, P_{E} \hat{n} g\right\rangle \\
{\left[B_{f, g}^{-}(E, t), \tilde{N}_{f^{\prime}, g^{\prime}}\left(E^{\prime}, t^{\prime}\right)\right]=} & 2 \pi \delta\left(t^{\prime}-t\right) \delta\left(E-E^{\prime}\right)\left\langle f, P_{E} f^{\prime}\right\rangle B_{g^{\prime}, g}^{-}(E, t) \\
{\left[\tilde{N}_{f, g}(E, t), \tilde{N}_{f^{\prime}, g^{\prime}}\left(E^{\prime}, t^{\prime}\right)\right]=} & 2 \pi \delta\left(t^{\prime}-t\right) \delta\left(E^{\prime}-E\right)\left[\left\langle g, P_{E} f^{\prime}\right\rangle N_{f, g^{\prime}}(E, t)\right. \\
& \left.-\left\langle g^{\prime}, P_{E} f\right\rangle N_{f^{\prime}, g}(E, t)\right]
\end{aligned}
$$

Suppose there exist such operators. Define $N_{f, g}(t):=\int \mathrm{d} E\left[\tilde{N}_{f, g}(E, t)+B_{g, f}^{-}(E, t)+\right.$ $\left.B_{f, g}^{+}(E, t)\right]$. Then one can prove exactly in the same way as in Theorem 6 that

$$
\lim _{\varepsilon \rightarrow 0} W_{\varepsilon, \hat{n},\left|f_{1}\right\rangle\left\langle g_{1}|, 0, \ldots,| f_{n}\right\rangle\left\langle g_{n}\right|, 0}\left(t_{1}, \ldots, t_{n}\right)=\left\langle\Omega, N_{f_{1}, g_{1}}\left(t_{1}\right) \ldots N_{f_{n}, g_{n}}\left(t_{n}\right) \Omega\right\rangle
$$




\section{ACKNOWLEDGEMENTS}

The author is grateful to Luigi Accardi for useful discussions and for kind hospitality in the Centro Vito Volterra of Rome University "Tor Vergata" where a significant part of this work was done. Special thanks to an anonymous referee for several suggestions improving the quality and content of the paper. The author acknowledges partial support from the grant RFFI-05-01-00884-a.

\section{APPENDIX A}

Here we make a connection between the objects defined in section II and the model of a test particle interacting with a dilute Bose gas (see Ref.$^{9}$ for details).

The one particle Hilbert space for this model has the form $\mathcal{H} \equiv L^{2}\left(\mathbb{R}^{3}\right)$, where $\mathbb{R}^{3}$ is the 3-dimensional coordinate or momentum space. The one particle free evolution is a unitary group $S_{t} \equiv \mathrm{e}^{\mathrm{i} t H_{1}}$ whose generator $H_{1}$ in the momentum representation is the multiplication operator by the function $\omega(\mathbf{k})=|\mathbf{k}|^{2} / 2 m$, where $m$ is the mass of a gas particle. The test particle is characterized by its Hilbert space $\mathcal{H}_{\mathrm{S}}$ and its free Hamiltonian $H_{\mathrm{S}}$ acting in $\mathcal{H}_{\mathrm{S}}$ which is assumed to have a discrete spectrum. The discrete set $B$ is the set of all transition frequencies of the test particle, or equivalently, the spectrum of its free Liouvillean $-\mathrm{i}\left[H_{\mathrm{S}}, \cdot\right]$.

The dynamics of a test particle interacting with a gas is described by an evolution operator $U(t)$ acting in $\mathcal{H}_{\mathrm{S}} \otimes \Gamma(\mathcal{H})$ and satisfying in the interaction picture, after the time rescaling $t \rightarrow t / \varepsilon$, the following Schrödinger equation

$$
\frac{\mathrm{d} U(t / \varepsilon)}{\mathrm{d} t}=-\mathrm{i}\left[\sum_{l, \omega} Q_{l, \omega} \otimes N_{T_{l}, \omega, \varepsilon}(t)\right] U(t / \varepsilon)
$$

Here $Q_{l, \omega}$ are certain operators in $\mathcal{H}_{\mathrm{S}}$ such that $\left[H_{\mathrm{S}}, Q_{l, \omega}\right]=-\omega Q_{l, \omega}$ and $T_{l}$ are certain operators in $\mathcal{H}$. The explicit form of these operators is determined by the details of the microscopic interaction between the test particle and particles of the gas. Equation (25) is the place where the operators $N_{T, \omega, \varepsilon}(t)$ appear.

The condition $S_{-t} \hat{n} S_{t}=\hat{n}$ and positivity of $\hat{n}$ imply that for this model $\hat{n}$ is a multiplication operator by a function $n: \mathbb{R}^{3} \rightarrow[0, \infty)$. The value $n(\mathbf{k})$ has the meaning

of the density of gas particles at momentum $\mathbf{k}$. If the state of the gas is $\omega_{\varepsilon \hat{n}}$ then the density of gas particles and the rate of collisions between the test particle and the gas are of order $\varepsilon$. Thus the limit $\varepsilon \rightarrow 0$ is the the low density limit. The limit is non-trivial since the dynamics is studied on the kinetic time scale of order $1 / \varepsilon$.

\section{APPENDIX B}

Let $S(\mathbb{R})$ be the Schwartz space over $\mathbb{R}$ and let $S^{\prime}(\mathbb{R})$ be the dual space of distributions. We reproduce the following lemma from ${ }^{4}$. 
Lemma 1 One has the limit in $S^{\prime}(\mathbb{R}) \times S^{\prime}(\mathbb{R})$

$$
\lim _{\varepsilon \rightarrow 0} \frac{\mathrm{e}^{\mathrm{i} t x / \varepsilon}}{\varepsilon}=2 \pi \delta(t) \delta(x)
$$

Proof. Let $f, \phi \in S(\mathbb{R})$ and let $\tilde{f}$ be Fourier transform of $f, \tilde{f}(\tau)=\int \mathrm{d} x \mathrm{e}^{\mathrm{i} \tau x} f(x)$. One has the identities

$I:=\lim _{\varepsilon \rightarrow 0} \int \mathrm{d} t \mathrm{~d} x \frac{\mathrm{e}^{\mathrm{i} t x / \varepsilon}}{\varepsilon} f(x) \phi(t)=\lim _{\varepsilon \rightarrow 0} \int \mathrm{d} \tau \phi(\varepsilon \tau) \int \mathrm{d} x \mathrm{e}^{\mathrm{i} \tau x} f(x)=\lim _{\varepsilon \rightarrow 0} \int \mathrm{d} \tau \phi(\varepsilon \tau) \tilde{f}(\tau)$

Since $\tilde{f} \in S(\mathbb{R})$, the function $\phi(\varepsilon \tau) \tilde{f}(\tau)$ satisfies the conditions of the Lebesgue lemma which allows to exchange the limit and integration in the last expression. Thus

$$
I=\phi(0) \int \mathrm{d} \tau \tilde{f}(\tau)=2 \pi \phi(0) f(0)
$$

${ }^{1}$ H. Spohn and J. L. Lebowitz, Adv. Chem. Phys. 38, 109 (1978).

${ }^{2}$ H. Spohn, Rev. Mod. Phys. 52, 569 (1980).

${ }^{3}$ R. Dümcke, Comm. Math. Phys. 97, 331 (1985).

${ }^{4}$ L. Accardi, Y.G. Lu, and I.V. Volovich, Quantum Theory and Its Stochastic Limit (Springer, Berlin, 2002).

${ }^{5}$ L. Accardi and Y. G. Lu, Comm. Math. Phys. 141, 9 (1991).

${ }^{6}$ S. Rudnicki, R. Alicki, and S. Sadowski, J. Math. Phys. 33, 2607 (1992).

7 L. Accardi, A. N. Pechen, and I.V. Volovich, J. Phys. A: Math. Gen. 35, 4889 (2002).

${ }^{8}$ L. Accardi, A. N. Pechen, and I. V. Volovich, Infinite Dimen. Anal., Quantum Probab., Relat. Top. 6, 431 (2003).

9 A. N. Pechen, J. Math. Phys. 45, 400 (2004).

10 D. V. Voiculescu, K. J. Dykema, and A. Nica, Free Random Variables CRM monograph series 1, American Mathematical Society, 1992.

11 D. Voiculescu, Lectures on Free Probability Theory, Lecture Notes in Math. 1738, 279-349 (Springer, Berlin, 2000).

${ }^{12}$ L. Accardi, Y.G. Lu, and V. Mastropietro, Infinite Dimen. Anal., Quantum Probab., Relat. Top. 1, 467 (1998).

${ }^{13}$ L. Accardi, I. Ya. Aref'eva, and I. V. Volovich, Proc. Steklov Inst. Math. 228, 106 (2000).

14 J. Andries, F. Benatti, M. De Cock, and M. Fannes, Rev. Math. Phys. 12, 921 (2000).

15 N. N. Bogoliubov, A. A. Logunov, A. I. Oksak, and I. T. Todorov, General Principles of Quantum Field Theory (Dordrecht, Boston, Kluwer Academic Publishers, 1990). 